\title{
LOCALLY DECOMPOSABLE GOLDEN RIEMANNIAN TANGENT BUNDLES WITH CHEEGER-GROMOLL METRIC
}

\author{
AHMET KAZAN AND H. BAYRAM KARADAĞ
}

Received 05 February, 2015

\begin{abstract}
In this paper we obtain a condition for the tangent bundle $(T M, \tilde{J}, \tilde{g})$ to be locally decomposable Golden Riemannian tangent bundle, where $\tilde{J}$ is the Golden structure on $T M$ and $\tilde{g}$ is the Cheeger-Gromoll metric.
\end{abstract}

2010 Mathematics Subject Classification: 53D10; 58A30

Keywords: horizontal lift, vertical lift, Cheeger-Gromoll metric, metallic structure

\section{INTRODUCTION}

The geometry of tangent bundle of a Riemannian manifold $(M, g)$ is very important in many areas of mathematics and physics. In recent years, a lot of studies about their local or global geometric properties have been published in the literature. When the authors studied this topic, they used different metrics which are called natural metrics on the tangent bundle. Firstly, the geometry of a tangent bundle has been studied by using a new metric $\hat{g}$, which is called Sasaki metric, with the aid of a Riemannian metric $g$ on a differential manifold $M$ in 1958 by Sasaki [15]. After that, the tangent bundle could be splitted to its horizontal and vertical subbundles with the aid of Levi-Civita connection $\nabla$ on $(M, g)$. Later, the Lie bracket of the tangent bundle $T M$, the Levi-Civita connection $\hat{\nabla}$ on $T M$ and its Riemannian curvature tensor $\hat{R}$ have been obtained in [3] and [10]. Furthermore, the explicit formulae of an another natural metric $\tilde{g}$, which is called Cheeger-Gromoll metric, on the tangent bundle $T M$ of a Riemannian manifold $(M, g)$ has been given by Musso and Tricerri in [13] by using the Cheeger and Gromoll's study [2]. The Levi-Civita connection $\tilde{\nabla}$ and the Riemannian curvature tensor $\tilde{R}$ of $(T M, \tilde{g})$ have been obtained in [16] and [6], respectively. Recently, the geometry of the tangent bundles with CheegerGromoll metric has been studied by many mathematicians (see [1,7,9,11,12,14] and etc.).

The differential geometry of the metallic means family (and usually the Golden mean) on Riemannian manifolds is a popular subject for mathematicians. In [4], Gezer et al. have studied the Riemannian manifolds endowed with Golden structures 
and they have given important theorems for these manifolds. In [8], some applications of the metallic means family and generalized Fibonacci sequences on Riemannian manifolds have been studied.

In this study, firstly we give the metallic structure $\tilde{J}$ on the tangent bundle $T M$ and obtain a integrability condition for $\tilde{J}$ with the aid of the Nijenhuis tensor field of $\tilde{J}$. Later, we find a condition for $(T M, \tilde{J}, \tilde{g})$ to be a metallic Riemannian manifold, where $\tilde{g}$ is the Cheeger-Gromoll metric on the tangent bundle $T M$. And finally, we give a characterization for locally decomposable Golden Riemannian tangent bundle $(T M, \tilde{J}, \tilde{g})$.

\section{Preliminaries}

Firstly, we shall give some notions about metallic Riemannian structures.

Fix two positive integers $p$ and $q$. The positive solution of the equation

$$
x^{2}-p x-q=0
$$

is named member of the metallic means family. These numbers, denoted

$$
\sigma_{p, q}=\frac{p+\sqrt{p^{2}+4 q}}{2}
$$

are also called $(p, q)$-metallic numbers ([8]).

Definition 1 ([8]). A polynomial structure on a manifold $M$ is called a metallic structure if it is determined by an $(1,1)$ tensor field $J$ which satisfies the equation

$$
J^{2}=p J+q I,
$$

where $p, q$ are positive integers and $I$ is the identity operator on the Lie algebra $\chi(M)$ of the vector fields on $M$.

We say that a Riemannian metric $g$ is $J$-compatible if

$$
g(J X, Y)=g(X, J Y)
$$

for every $X, Y \in \chi(M)$, which means that $J$ is a self-adjoint operator with respect to $g$. For metallic structure, this condition is equivalent with

$$
g(J X, J Y)=p \cdot g(X, J Y)+q \cdot g(X, Y)([8]) .
$$

Definition 2 ([8]). A Riemannian manifold $(M, g)$ endowed with a metallic structure $J$ so that the Riemannian metric $g$ is $J$-compatible is named a metallic Riemannian manifold and $(g, J)$ is called a metallic Riemannian structure on $M$.

Remark 1. If we consider $p=q=1$ in the above definition, then we obtain that $(g, J)$ is a golden Riemannian structure of $M$ and similarly,

if $p=2$ and $q=1$, then $(g, J)$ is a silver Riemannian structure of $M$;

if $p=3$ and $q=1$, then $(g, J)$ is a bronze Riemannian structure of $M$;

if $p=4$ and $q=1$, then $(g, J)$ is a subtle Riemannian structure of $M$; 
if $p=1$ and $q=2$, then $(g, J)$ is a copper Riemannian structure of $M$ and

if $p=1$ and $q=3$, then $(g, J)$ is a nickel Riemannian structure of $M$ (for detail, see $[8])$.

Let $J$ be a $(1,1)$-tensor field on $M$, i.e. $J \in \mathfrak{F}_{1}^{1}(M)$. A tensor field $t$ of type $(\mathrm{r}, \mathrm{s})$ is called a pure tensor field with respect to $J$ if

$$
\begin{aligned}
t\left(J X_{1}, X_{2}, \ldots, X_{s} ; \xi^{1}, \xi^{2}, \ldots, \xi^{r}\right) & =t\left(X_{1}, J X_{2}, \ldots, X_{s} ; \xi^{1}, \xi^{2}, \ldots, \xi^{r}\right) \\
& \vdots \\
& =t\left(X_{1}, X_{2}, \ldots, J X_{s} ; \xi^{1}, \xi^{2}, \ldots, \xi^{r}\right) \\
& =t\left(X_{1}, X_{2}, \ldots, X_{s} ; J^{\prime} \xi^{1}, \xi^{2}, \ldots, \xi^{r}\right) \\
& \vdots \\
& =t\left(X_{1}, X_{2}, \ldots, X_{s} ; \xi^{1}, \xi^{2}, \ldots, J^{\prime} \xi^{r}\right),
\end{aligned}
$$

for any $X_{1}, X_{2}, \ldots, X_{r} \in \mathfrak{F}_{0}^{1}(M)$ and $\xi^{1}, \xi^{2}, \ldots, \xi^{r} \in \mathfrak{F}_{1}^{0}(M)$, where $J^{\prime}$ is the adjoint operator of $J$ defined by

$$
\left(J^{\prime} \xi\right)(X)=\xi(J X)=(\xi \circ J)(X), X \in \mathfrak{F}_{0}^{1}(M), \xi \in \mathfrak{F}_{1}^{0}(M) .
$$

So, the $J$-compatible Riemannian metric $g$ is a pure tensor field.

We define an operator

$$
\Phi_{J}: \mathfrak{F}_{s}^{0}(M) \rightarrow \mathfrak{F}_{s+1}^{0}(M)
$$

applied to the pure tensor field $t$ of type $(0, \mathrm{~s})$ with respect to $J$ by

$$
\begin{aligned}
\left(\Phi_{J} t\right)\left(X, Y_{1}, \ldots, Y_{S}\right)= & (J X) t\left(Y_{1}, \ldots, Y_{s}\right)-X t\left(J Y_{1}, \ldots, Y_{S}\right) \\
& +\sum_{\lambda=1}^{s} t\left(Y_{1}, \ldots,\left(L_{Y_{\lambda}} J\right) X, \ldots, Y_{S}\right),
\end{aligned}
$$

for any $X, Y_{1}, \ldots, Y_{S} \in \mathfrak{F}_{0}^{1}(M)$, where $L_{Y}$ denotes the Lie differentiation with respect to $Y$ (for detail, see [4]).

Proposition 1 ([8]). Every almost product structure F induces two metallic structures on $M$ given as follows:

$$
J_{1}=\frac{p}{2} I+\left(\frac{2 \sigma_{p, q}-p}{2}\right) F, \quad J_{2}=\frac{p}{2} I-\left(\frac{2 \sigma_{p, q}-p}{2}\right) F .
$$

Conversely, every metallic structure $J$ on $M$ induces two almost product structures on this manifold:

$$
F=\mp\left(\frac{2}{2 \sigma_{p, q}-p} J-\frac{p}{2 \sigma_{p, q}-p} I\right) .
$$

In particular, if the almost product structure $F$ is a Riemannian one, then $J_{1}, J_{2}$ are also metallic Riemannian structures. 
Remark 2. i) If $J$ is a golden structure on $M$, then every almost product structure $F$ induces two golden structures on $M$ given by

$$
J_{1,2}=\frac{1}{2}(I \mp \sqrt{5} F)
$$

and conversely, every golden structure $J$ on $M$ induces two almost product structures on $M$ given by

$$
F=\mp \frac{1}{\sqrt{5}}(2 J-I) \text {. }
$$

Similarly,

ii) every almost product structure $F$ induces two silver structures on $M$ given by

$$
J_{1,2}=I \mp \sqrt{2} F
$$

and conversely, every silver structure $J$ on $M$ induces two almost product structures on this manifold by

$$
F=\mp \frac{1}{\sqrt{2}}(J-I)
$$

iii) every almost product structure $F$ induces two bronze structures on $M$ given by

$$
J_{1,2}=\frac{1}{2}(3 I \mp \sqrt{13} F)
$$

and conversely, every bronze structure $J$ on $M$ induces two almost product structures on this manifold by

$$
F=\mp \frac{1}{\sqrt{13}}(2 J-3 I) ;
$$

iv) every almost product structure $F$ induces two subtle structures on $M$ given by

$$
J_{1,2}=2 I \mp \sqrt{5} F
$$

and conversely, every subtle structure $J$ on $M$ induces two almost product structures on this manifold by

$$
F=\mp \frac{1}{\sqrt{5}}(J-2 I) ;
$$

v) every almost product structure $F$ induces two copper structures on $M$ given by

$$
J_{1,2}=\frac{1}{2}(I \mp 3 F)
$$

and conversely, every copper structure $J$ on $M$ induces two almost product structures on this manifold by

$$
F=\mp \frac{1}{3}(2 J-I)
$$

vi) every almost product structure $F$ induces two nickel structures on $M$ given by

$$
J_{1,2}=\frac{1}{2}(I \mp \sqrt{13} F)
$$


and conversely, every nickel structure $J$ on $M$ induces two almost product structures on this manifold by

$$
F=\mp \frac{1}{\sqrt{13}}(2 J-I) .
$$

Here, we shall recall lifts of vector fields on Riemannian manifolds to their tangent bundles.

Let $\nabla$ be the Levi-Civita connection of the Riemannian metric $g$ on $M$ and the differential of the bundle map $\pi: T M \longrightarrow M$ be the smooth map $d \pi: T T M \longrightarrow$ $T M$. Then,

Proposition 2 ([3]). The tangent space $T_{(p, u)} T M$ at a point $(p, u)$ in $T M$ is a direct sum of its horizontal and vertical subspaces, i.e.

$$
T_{(p, u)} T M=\mathscr{H}_{(p, u)} \oplus \mathcal{V}_{(p, u)} .
$$

The horizontal and vertical lifts of tangent vectors on $M$ are defined as follows:

Definition 3 ([5]). If $X \in T_{p} M$ is a tangent vector, then the horizontal lift of $X$ to a point $(p, u) \in T M$ is the unique vector $X^{h} \in \mathscr{H}_{(p, u)}$ such that $d \pi\left(X^{h}\right)=X$. The vertical lift of $X$ to $(p, u)$ is the unique vector $X^{v} \in \mathcal{V}_{(p, u)}$ such that $X^{v}(d f)=X(f)$ for all functions $f$ on $M$, where $d f$ is the function defined by $(d f)(p, u)=u(f)$.

Now, we can extend the notions of horizontal and vertical lifts to vector fields.

Definition 4 ([5]). The horizontal lift of a vector field $X \in C^{\infty}(T M)$ is the vector field $X^{h} \in C^{\infty}(T T M)$ whose value at each point $(p, u)$ is the horizontal lift of $X(p)$ to $(p, u)$. The vertical lift of $X \in C^{\infty}(T M)$ to $T M$ is the vector field $X^{v} \in$ $C^{\infty}(T T M)$ whose value at each point $(p, u)$ is the vertical lift of $X(p)$ to $(p, u)$.

Note that, the map $X \longrightarrow X^{h}$ is an isomorphism between the vector spaces $T_{p} M$ and $\mathscr{H}_{(p, u)}$ and similarly the map $X \longrightarrow X^{v}$ is an isomorphism between the vector spaces $T_{p} M$ and $\mathcal{V}_{(p, u)}$. Obviously, each tangent vector $\tilde{Z} \in T_{(p, u)} T M$ can be written in the form $\tilde{Z}=X^{h}+Y^{v}$, where $X, Y \in T_{p} M$ are uniquely determined vectors. If $\varphi$ is a smooth function on $M$, then

$$
X^{h}(\varphi \circ \pi)=(X \varphi) \circ \pi \text { and } X^{v}(\varphi \circ \pi)=0
$$

hold for all vector fields $X$ on $M$ [5].

For local coordinates $(V, x)$ on an $n$-dimensional manifold $M$, we define the smooth functions

$$
v_{1}, v_{2}, \ldots, v_{2 n}: T M \longrightarrow \mathbb{R}
$$

by

$$
\begin{aligned}
& v_{i}=x_{i} \circ \pi \\
& v_{n+i}(Y)=Y\left(x_{i}\right)=d x_{i}(Y)
\end{aligned}
$$


for all $Y \in T M$ and $i=1,2, \ldots, n$. Then, $\left(v_{1}, v_{2}, \ldots, v_{2 n}\right): \pi^{-1}(V) \subset T M \longrightarrow \mathbb{R}^{2 n}$ are local coordinates on $T M$. Then,

Lemma 1 ([3]). Let $(M, g)$ be a Riemannian manifold and $X, Z \in C^{\infty}(T M)$ be vector fields on $M$ which are locally represented by

$$
X=\sum_{i=1}^{n} \xi_{i} \frac{\partial}{\partial x_{i}} \text { and } Z=\sum_{i=1}^{n} \eta_{i} \frac{\partial}{\partial x_{i}} .
$$

Then, vertical and horizontal lifts $X^{v}$ and $X^{h}$ of $X$ at the point $Z \in T M$ are given by

$$
\begin{gathered}
\left(X^{v}\right)_{Z}=\sum_{i=1}^{n} \xi_{i} \frac{\partial}{\partial v_{n+i}}, \\
\left(X^{h}\right)_{Z}=\sum_{i=1}^{n} \xi_{i} \frac{\partial}{\partial v_{i}}-\left(\sum_{i, j, k=1}^{n} \xi_{j} \eta_{k} \Gamma_{j k}^{i}\right) \frac{\partial}{\partial v_{n+i}},
\end{gathered}
$$

respectively, where the coefficients $\Gamma_{j k}^{i}$ are the Christoffel symbols of the Levi-Civita connection $\nabla$ on $(M, g)$.

Proposition 3 ([3]). Let $(M, g)$ be a Riemannian manifold with Levi-Civita connection $\nabla$ and Riemannian curvature tensor $R$ which is defined by $R(X, Y)=$ $\left[\nabla_{X}, \nabla_{Y}\right]-\nabla_{[X, Y]}$. Then the Lie bracket of the tangent bundle TM of $M$ satisfies the following:

i) $\left[X^{v}, Y^{v}\right]=0$,

ii) $\left[X^{h}, Y^{v}\right]=\left(\nabla_{X} Y\right)^{v}$,

iii) $\left[X^{h}, Y^{h}\right]=[X, Y]^{h}-(R(X, Y) u)^{v}$, for all vector fields $X, Y \in C^{\infty}(T M)$ and $(p, u) \in T M$.

Here, we shall recall the notion of a natural class of metrics on the tangent bundle $T M$ of a given Riemannian manifold $(M, g)$.

Definition 5 ([5]). Let $(M, g)$ be a Riemannian manifold. A Riemannian metric $\bar{g}$ on the tangent bundle $T M$ is said to be natural with respect to $g$ on $M$ if

i) $\bar{g}_{(p, u)}\left(X^{h}, Y^{h}\right)=g_{p}(X, Y)$,

ii) $\bar{g}_{(p, u)}\left(X^{h}, Y^{v}\right)=0$, for all vector fields $X, Y \in C^{\infty}(T M)$ and $(p, u) \in T M$.

The Cheeger-Gromoll metric is a positive definite natural metric on $T M$ which is described in terms of lifted vectors as follows:

Definition 6 ([5]). Let $g$ be a Riemannian metric on a manifold $M$. Then, the Cheeger-Gromoll (C-G) metric is a Riemannian metric $\tilde{g}$ on the tangent bundle $T M$ such that

i) $\tilde{g}_{(p, u)}\left(X^{h}, Y^{h}\right)=g_{p}(X, Y)$, 
ii) $\tilde{g}_{(p, u)}\left(X^{h}, Y^{v}\right)=0$,

iii) $\tilde{g}_{(p, u)}\left(X^{v}, Y^{v}\right)=\frac{1}{1+r^{2}}\left\{g_{p}(X, Y)+g_{p}(X, u) g_{p}(Y, u)\right\}$, for all $X, Y \in C^{\infty}(T M)$. Here $r$ denotes $|u|=\sqrt{g(u, u)}$.

Furthermore, for the Cheeger-Gromoll metric $\tilde{g}$, we have

$$
\left.\begin{array}{rl}
X^{h}\left(\tilde{g}\left(Y^{h}, Z^{h}\right)\right)= & X(g(Y, Z)), \\
X^{v}\left(\tilde{g}\left(Y^{h}, Z^{h}\right)\right)= & 0 \\
X^{h}\left(\tilde{g}\left(Y^{v}, Z^{v}\right)\right)= & \tilde{g}\left(\left(\nabla_{X} Y\right)^{v}, Z^{v}\right)+\tilde{g}\left(Y^{v},\left(\nabla_{X} Z\right)^{v}\right), \\
X^{v}\left(\tilde{g}\left(Y^{v}, Z^{v}\right)\right)= & -\frac{2}{\alpha^{2}} g(X, u)\{g(Y, Z)+g(Y, u) g(Z, u)\} \\
& +\frac{1}{\alpha}\{g(X, Y) g(Z, u)+g(X, Z) g(Y, u)\},
\end{array}\right\}
$$

(for detail, see [5]).

\section{LOCALLY DECOMPOSABLE GOLDEN TANGENT BUNDLES WITH \\ CHEEGER-GROMOLL METRIC}

A metallic structure $\tilde{J}$ on the tangent bundle $T M$ is defined by

$$
\left.\begin{array}{l}
\tilde{J} X^{h}=\frac{1}{2}\left\{p X^{h}+\left(2 \sigma_{p, q}-p\right) X^{v}\right\} \\
\tilde{J} X^{v}=\frac{1}{2}\left\{p X^{v}+\left(2 \sigma_{p, q}-p\right) X^{h}\right\},
\end{array}\right\}
$$

for all vector fields $X$ and $Y$. Here one can easily see that, the structure $\tilde{J}$ in (3.1) satisfies the condition $\tilde{J}^{2}-p \tilde{J}-q I=0$, actually. So, we can note that

Remark 3. The golden structure $\tilde{J}$ on the tangent bundle $T M$ is defined by

$$
\left.\begin{array}{c}
\tilde{J} X^{h}=\frac{1}{2}\left\{X^{h}+\sqrt{5} X^{v}\right\} \\
\tilde{J} X^{v}=\frac{1}{2}\left\{X^{v}+\sqrt{5} X^{h}\right\},
\end{array}\right\}
$$

the silver structure $\tilde{J}$ on the tangent bundle $T M$ is defined by

$$
\left.\begin{array}{c}
\tilde{J} X^{h}=X^{h}+\sqrt{2} X^{v} \\
\tilde{J} X^{v}=X^{v}+\sqrt{2} X^{h}
\end{array}\right\}
$$

the bronze structure $\tilde{J}$ on the tangent bundle $T M$ is defined by

$$
\left.\begin{array}{c}
\tilde{J} X^{h}=\frac{1}{2}\left\{3 X^{h}+\sqrt{13} X^{v}\right\} \\
\tilde{J} X^{v}=\frac{1}{2}\left\{3 X^{v}+\sqrt{13} X^{h}\right\},
\end{array}\right\}
$$

the subtle structure $\tilde{J}$ on the tangent bundle $T M$ is defined by

$$
\left.\begin{array}{l}
\tilde{J} X^{h}=2 X^{h}+\sqrt{5} X^{v} \\
\tilde{J} X^{v}=2 X^{v}+\sqrt{5} X^{h}
\end{array}\right\}
$$

the copper structure $\tilde{J}$ on the tangent bundle $T M$ is defined by

$$
\left.\begin{array}{l}
\tilde{J} X^{h}=\frac{1}{2}\left\{X^{h}+3 X^{v}\right\} \\
\tilde{J} X^{v}=\frac{1}{2}\left\{X^{v}+3 X^{h}\right\}
\end{array}\right\}
$$


and the nickel structure $\tilde{J}$ on the tangent bundle $T M$ is defined by

$$
\left.\begin{array}{l}
\tilde{J} X^{h}=\frac{1}{2}\left\{X^{h}+\sqrt{13} X^{v}\right\} \\
\tilde{J} X^{v}=\frac{1}{2}\left\{X^{v}+\sqrt{13} X^{h}\right\} .
\end{array}\right\}
$$

Let $N_{\tilde{J}}(\tilde{X}, \tilde{Y})$ be the Nijenhuis torsion tensor field of the metallic structure $\tilde{J}$, defined by

$$
N_{\tilde{J}}(\tilde{X}, \tilde{Y})=[\tilde{J} \tilde{X}, \tilde{J} \tilde{Y}]+\tilde{J}^{2}[\tilde{X}, \tilde{Y}]-\tilde{J}[\tilde{J} \tilde{X}, \tilde{Y}]-\tilde{J}[\tilde{X}, \tilde{J} \tilde{Y}]
$$

for all $\tilde{X}, \tilde{Y} \in T_{(p, u)} T M$.

From (2.1), (3.1), (3.8) and Proposition (3), we get

$$
\begin{aligned}
N_{\tilde{J}} & \left(X^{h}, Y^{h}\right)=\left[\tilde{J} X^{h}, \tilde{J} Y^{h}\right]+\tilde{J}^{2}\left[X^{h}, Y^{h}\right]-\tilde{J}\left[\tilde{J} X^{h}, Y^{h}\right]-\tilde{J}\left[X^{h}, \tilde{J} Y^{h}\right] \\
= & {\left[\frac{p}{2} X^{h}+\left(\frac{2 \sigma_{p, q}-p}{2}\right) X^{v}, \frac{p}{2} Y^{h}+\left(\frac{2 \sigma_{p, q}-p}{2}\right) Y^{v}\right]+\tilde{J}^{2}\left[X^{h}, Y^{h}\right] } \\
& -\tilde{J}\left[\frac{p}{2} X^{h}+\left(\frac{2 \sigma_{p, q}-p}{2}\right) X^{v}, Y^{h}\right]-\tilde{J}\left[X^{h}, \frac{p}{2} Y^{h}+\left(\frac{2 \sigma_{p, q}-p}{2}\right) Y^{v}\right] \\
= & \frac{p^{2}}{4}\left[X^{h}, Y^{h}\right]+\frac{p}{2}\left(\frac{2 \sigma_{p, q}-p}{2}\right)\left(\nabla_{X} Y\right)^{v}-\frac{p}{2}\left(\frac{2 \sigma_{p, q}-p}{2}\right)\left(\nabla_{Y} X\right)^{v} \\
& +p \tilde{J}[X, Y]^{h}-p \tilde{J}(R(X, Y) u)^{v}+q[X, Y]^{h}-q(R(X, Y) u)^{v}-\frac{p}{2} \tilde{J}[X, Y]^{h} \\
& +\frac{p}{2} \tilde{J}(R(X, Y) u)^{v}+\left(\frac{2 \sigma_{p, q}-p}{2}\right) \tilde{J}\left(\nabla_{Y} X\right)^{v}-\frac{p}{2} \tilde{J}[X, Y]^{h} \\
& +\frac{p}{2} \tilde{J}(R(X, Y) u)^{v}-\left(\frac{2 \sigma_{p, q}-p}{2}\right) \tilde{J}\left(\nabla_{X} Y\right)^{v} \\
= & \left(\frac{p^{2}}{4}+q-\left(\frac{2 \sigma_{p, q}-p}{2}\right)^{2}\right)[X, Y]^{h}-\left(\frac{p^{2}}{4}+q\right)(R(X, Y) u)^{v} \\
= & -\left(\frac{p^{2}+4 q}{4}\right)(R(X, Y) u)^{v}
\end{aligned}
$$

and similarly

$$
\begin{aligned}
N_{\tilde{J}}\left(X^{h}, Y^{v}\right)= & {\left[\tilde{J} X^{h}, \tilde{J} Y^{v}\right]+\tilde{J}^{2}\left[X^{h}, Y^{v}\right]-\tilde{J}\left[\tilde{J} X^{h}, Y^{v}\right]-\tilde{J}\left[X^{h}, \tilde{J} Y^{v}\right] } \\
= & {\left[\frac{p}{2} X^{h}+\left(\frac{2 \sigma_{p, q}-p}{2}\right) X^{v}, \frac{p}{2} Y^{v}+\left(\frac{2 \sigma_{p, q}-p}{2}\right) Y^{h}\right]+\tilde{J}^{2}\left(\nabla_{X} Y\right)^{v} } \\
& -\tilde{J}\left[\frac{p}{2} X^{h}+\left(\frac{2 \sigma_{p, q}-p}{2}\right) X^{v}, Y^{v}\right]-\tilde{J}\left[X^{h}, \frac{p}{2} Y^{v}+\left(\frac{2 \sigma_{p, q}-p}{2}\right) Y^{h}\right] \\
= & \left(\frac{2 \sigma_{p, q}-p}{2}\right)^{2}(R(X, Y) u)^{h} \\
= & \left(\frac{p^{2}+4 q}{4}\right)(R(X, Y) u)^{h},
\end{aligned}
$$




$$
\begin{aligned}
N_{\tilde{J}}\left(X^{v}, Y^{h}\right)= & {\left[\tilde{J} X^{v}, \tilde{J} Y^{h}\right]+\tilde{J}^{2}\left[X^{v}, Y^{h}\right]-\tilde{J}\left[\tilde{J} X^{v}, Y^{h}\right]-\tilde{J}\left[X^{v}, \tilde{J} Y^{h}\right] } \\
= & {\left[\frac{p}{2} X^{v}+\left(\frac{2 \sigma_{p, q}-p}{2}\right) X^{h}, \frac{p}{2} Y^{h}+\left(\frac{2 \sigma_{p, q}-p}{2}\right) Y^{v}\right]-\tilde{J}^{2}\left(\nabla_{Y} X\right)^{v} } \\
& -\tilde{J}\left[\frac{p}{2} X^{v}+\left(\frac{2 \sigma_{p, q}-p}{2}\right) X^{h}, Y^{h}\right]-\tilde{J}\left[X^{v}, \frac{p}{2} Y^{h}+\left(\frac{2 \sigma_{p, q}-p}{2}\right) Y^{v}\right] \\
= & \left(\frac{2 \sigma_{p, q}-p}{2}\right)^{2}(R(X, Y) u)^{h} \\
= & \left(\frac{p^{2}+4 q}{4}\right)(R(X, Y) u)^{h}
\end{aligned}
$$

and

$$
\begin{aligned}
N_{\tilde{J}}\left(X^{v}, Y^{v}\right)= & {\left[\tilde{J} X^{v}, \tilde{J} Y^{v}\right]+\tilde{J}^{2}\left[X^{v}, Y^{v}\right]-\tilde{J}\left[\tilde{J} X^{v}, Y^{v}\right]-\tilde{J}\left[X^{v}, \tilde{J} Y^{v}\right] } \\
= & {\left[\frac{p}{2} X^{v}+\left(\frac{2 \sigma_{p, q}-p}{2}\right) X^{h}, \frac{p}{2} Y^{v}+\left(\frac{2 \sigma_{p, q}-p}{2}\right) Y^{h}\right] } \\
& -\tilde{J}\left[\frac{p}{2} X^{v}+\left(\frac{2 \sigma_{p, q}-p}{2}\right) X^{h}, Y^{v}\right]-\tilde{J}\left[X^{v}, \frac{p}{2} Y^{v}+\left(\frac{2 \sigma_{p, q}-p}{2}\right) Y^{h}\right] \\
= & -\left(\frac{2 \sigma_{p, q}-p}{2}\right)^{2}(R(X, Y) u)^{v} \\
= & -\left(\frac{p^{2}+4 q}{4}\right)(R(X, Y) u)^{v},
\end{aligned}
$$

where $\mathrm{R}(\mathrm{X}, \mathrm{Y})$ means the Riemannian curvature tensor of the Riemannian manifold $M$.

Therefore, from (3.9)-(3.12), we have the following theorem:

Theorem 1. The metallic structure $\tilde{J}$ on the tangent bundle TM is integrable if and only if $M$ is flat, i.e. $R \equiv 0$.

Proof. If $\tilde{J}$ is integrable, then $N \equiv 0$. So from (3.9)-(3.12), we have $R \equiv 0$. Conversely, if $M$ is flat, i.e. $R \equiv 0$, then we get $N \equiv 0$ from(3.9)-(3.12). So, this completes the proof.

Let we define,

$$
\tilde{G}(\tilde{X}, \tilde{Y})=\tilde{g}(\tilde{J} \tilde{X}, \tilde{Y})-\tilde{g}(\tilde{X}, \tilde{J} \tilde{Y}), \quad \tilde{X}, \tilde{Y} \in T_{(p, u)} T M
$$

From the definition of the Cheeger-Gromoll metric and (3.1), we have

$$
\begin{aligned}
\tilde{G}\left(X^{h}, Y^{h}\right)= & \tilde{g}\left(\tilde{J} X^{h}, Y^{h}\right)-\tilde{g}\left(X^{h}, \tilde{J} Y^{h}\right) \\
= & \tilde{g}\left(\frac{1}{2}\left\{p X^{h}+\left(2 \sigma_{p, q}-p\right) X^{v}\right\}, Y^{h}\right) \\
& -\tilde{g}\left(X^{h}, \frac{1}{2}\left\{p Y^{h}+\left(2 \sigma_{p, q}-p\right) Y^{v}\right\}\right)
\end{aligned}
$$




$$
\begin{aligned}
= & \frac{1}{2}\left\{p \tilde{g}\left(X^{h}, Y^{h}\right)+\left(2 \sigma_{p, q}-p\right) \tilde{g}\left(X^{v}, Y^{h}\right)\right. \\
& \left.-p \tilde{g}\left(X^{h}, Y^{h}\right)-\left(2 \sigma_{p, q}-p\right) \tilde{g}\left(X^{h}, Y^{v}\right)\right\} \\
= & 0
\end{aligned}
$$

$$
\begin{aligned}
\tilde{G}\left(X^{h}, Y^{v}\right)= & \tilde{g}\left(\tilde{J} X^{h}, Y^{v}\right)-\tilde{g}\left(X^{h}, \tilde{J} Y^{v}\right) \\
= & \tilde{g}\left(\frac{1}{2}\left\{p X^{h}+\left(2 \sigma_{p, q}-p\right) X^{v}\right\}, Y^{v}\right) \\
& -\tilde{g}\left(X^{h}, \frac{1}{2}\left\{p Y^{v}+\left(2 \sigma_{p, q}-p\right) Y^{h}\right\}\right) \\
= & \left(\frac{2 \sigma_{p, q}-p}{2}\right)\left\{\tilde{g}\left(X^{v}, Y^{v}\right)-\tilde{g}\left(X^{h}, Y^{h}\right)\right\} \\
= & \left(\frac{2 \sigma_{p, q}-p}{2}\right)\left\{\frac{g(X, u) g(Y, u)-g(X, Y) g(u, u)}{1+g(u, u)}\right\}, \\
\tilde{G}\left(X^{v}, Y^{h}\right)= & \tilde{g}\left(\tilde{J} X^{v}, Y^{h}\right)-\tilde{g}\left(X^{v}, \tilde{J} Y^{h}\right) \\
= & \tilde{g}\left(\frac{1}{2}\left\{p X^{v}+\left(2 \sigma_{p, q}-p\right) X^{h}\right\}, Y^{h}\right) \\
& -\tilde{g}\left(X^{v}, \frac{1}{2}\left\{p Y^{h}+\left(2 \sigma_{p, q}-p\right) Y^{v}\right\}\right) \\
= & \left(\frac{2 \sigma_{p, q}-p}{2}\right)\left\{\tilde{g}\left(X^{h}, Y^{h}\right)-\tilde{g}\left(X^{v}, Y^{v}\right)\right\} \\
= & \left(\frac{2 \sigma_{p, q}-p}{2}\right)\left\{\frac{g(X, Y) g(u, u)-g(X, u) g(Y, u)}{1+g(u, u)}\right\}
\end{aligned}
$$

and

$$
\begin{aligned}
\tilde{G}\left(X^{v}, Y^{v}\right)= & \tilde{g}\left(\tilde{J} X^{v}, Y^{v}\right)-\tilde{g}\left(X^{v}, \tilde{J} Y^{v}\right) \\
= & \tilde{g}\left(\frac{1}{2}\left\{p X^{v}+\left(2 \sigma_{p, q}-p\right) X^{h}\right\}, Y^{v}\right) \\
& -\tilde{g}\left(X^{v}, \frac{1}{2}\left\{p Y^{v}+\left(2 \sigma_{p, q}-p\right) Y^{h}\right\}\right) \\
= & \frac{1}{2}\left\{p \tilde{g}\left(X^{v}, Y^{v}\right)+\left(2 \sigma_{p, q}-p\right) \tilde{g}\left(X^{h}, Y^{v}\right)\right. \\
& \left.-p \tilde{g}\left(X^{v}, Y^{v}\right)-\left(2 \sigma_{p, q}-p\right) \tilde{g}\left(X^{v}, Y^{h}\right)\right\} \\
= & 0 .
\end{aligned}
$$

So, we have

Theorem 2. Let $(M, g)$ be a Riemannian manifold and TM be its tangent bundle equipped with the Cheeger-Gromoll metric $\tilde{g}$ and the metallic structure $\tilde{J}$ defined 
by (3.1). Then, $(T M, \tilde{J}, \tilde{g})$ is a metallic Riemannian tangent bundle if and only if $g(X, u) u=g(u, u) X$ holds on $M$ for all vector fields $X$.

Proof. The proof is obvious from (2.2), (3.13)-(3.17) and Definition (2).

Corollary 1. Let $(M, g)$ be a Riemannian manifold and TM be its tangent bundle equipped with the Cheeger-Gromoll metric $\tilde{g}$ and the metallic structure $\tilde{J}$ defined by (3.1). Then, $\tilde{g}$ is pure with respect to the metallic structure $\tilde{J}$ if and only if $g(X, u) u=$ $g(u, u) X$ holds on $M$ for all vector fields $X$.

Now, we shall give a characterization for Golden Riemannian manifold which is a class of metallic Riemannian manifolds (i.e. $p=q=1$ in (2.1)).

A Golden Riemannian manifold $(M, J, g)$ with an integrable Golden structure $J$ is called a locally Golden Riemannian manifold. If the metric $g$ of the locally Golden Riemannian manifold has the form

$d s^{2}=g_{a b}\left(x^{c}\right) d x^{a} d x^{b}+g_{\bar{a} \bar{b}}\left(x^{\bar{c}}\right) d x^{\bar{a}} d x^{\bar{b}}, a, b, c=1, \ldots, m, \bar{a}, \bar{b}, \bar{c}=m+1, \ldots, n$, that is $g_{a b}$ are functions of $x^{c}$ only, $g_{a \bar{b}}=0$ and $g_{\bar{a} \bar{b}}$ are functions of $x^{\bar{c}}$ only, then we call the manifold $M$ a locally decomposable Golden Riemannian manifold [4].

In [4], Gezer et al. have seen that, a Golden Riemannian manifold $(M, J, g)$ is locally decomposable Golden Riemannian manifold if and only if $\Phi_{F} g=0$, where $F$ is the corresponding almost product structure. And they have had a relation between the corresponding almost product structure $F$ and Golden structure $J$ as follows:

$$
\Phi_{F} g=\frac{2}{\sqrt{5}} \Phi_{J} g .
$$

So, from these conclusions, we have the following theorem:

Theorem 3. Let $(M, g)$ be a Riemannian manifold and TM be its tangent bundle equipped with the Cheeger-Gromoll metric $\tilde{g}$ and the Golden structure $\tilde{J}$ defined by (3.2). Then, $(T M, \tilde{J}, \tilde{g})$ is a locally decomposable Golden Riemannian tangent bundle if and only if $g(X, Y) u+g(Y, u) X=2 g(X, u) Y$ holds on $M$ for all vector fields $X$ and $Y$.

Proof. From the definition of the Cheeger-Gromoll metric, (2.4), (2.5) and (3.2) we get

$$
\begin{aligned}
& \left(\Phi_{\tilde{J}} \tilde{g}\right)\left(X^{h}, Y^{h}, Z^{h}\right) \\
& =\left(\tilde{J} X^{h}\right)\left(\tilde{g}\left(Y^{h}, Z^{h}\right)\right)-X^{h}\left(\tilde{g}\left(\tilde{J} Y^{h}, Z^{h}\right)\right) \\
& \quad+\tilde{g}\left(\left[Y^{h}, \tilde{J} X^{h}\right]-\tilde{J}\left[Y^{h}, X^{h}\right], Z^{h}\right)+\tilde{g}\left(Y^{h},\left[Z^{h}, \tilde{J} X^{h}\right]-\tilde{J}\left[Z^{h}, X^{h}\right]\right) \\
& =\left(\frac{1}{2} X^{h}+\frac{\sqrt{5}}{2} X^{v}\right)\left(\tilde{g}\left(Y^{h}, Z^{h}\right)\right)-X^{h}\left(\tilde{g}\left(\frac{1}{2} Y^{h}+\frac{\sqrt{5}}{2} Y^{v}, Z^{h}\right)\right) \\
& \quad+\tilde{g}\left(\left[Y^{h}, \frac{1}{2} X^{h}+\frac{\sqrt{5}}{2} X^{v}\right]-\tilde{J}\left[Y^{h}, X^{h}\right], Z^{h}\right)
\end{aligned}
$$




$$
\begin{aligned}
& +\tilde{g}\left(Y^{h},\left[Z^{h}, \frac{1}{2} X^{h}+\frac{\sqrt{5}}{2} X^{v}\right]-\tilde{J}\left[Z^{h}, X^{h}\right]\right) \\
= & \tilde{g}\left(\frac{1}{2}[Y, X]^{h}-\frac{1}{2}(R(Y, X) u)^{v}+\frac{\sqrt{5}}{2}\left(\nabla_{Y} X\right)^{v}-\tilde{J}[Y, X]^{h}\right. \\
& \left.+\tilde{J}(R(Y, X) u)^{v}, Z^{h}\right)+\tilde{g}\left(Y^{h}, \frac{1}{2}[Z, X]^{h}-\frac{1}{2}(R(Z, X) u)^{v}\right. \\
& +\frac{\sqrt{5}}{2}\left(\nabla_{Z} X\right)^{v}-\tilde{J}[Z, X]^{h}+\tilde{J}(R(Z, X) u)^{v} \\
= & \tilde{g}\left(\frac{\sqrt{5}}{2}(R(Y, X) u)^{h}, Z^{h}\right)+\tilde{g}\left(Y^{h}, \frac{\sqrt{5}}{2}(R(Z, X) u)^{h}\right) \\
= & \frac{\sqrt{5}}{2} g(R(Y, X) u-R(u, Y) X, Z) .
\end{aligned}
$$

With similar calculations, we have

$$
\begin{aligned}
\left(\Phi_{\tilde{J}} \tilde{g}\right)\left(X^{h}, Y^{h}, Z^{v}\right)= & \frac{\sqrt{5}}{2(1+g(u, u))}\left\{g\left(Y, \nabla_{X} Z\right) g(u, u)-g(Y, u) g\left(\nabla_{X} Z, u\right)\right\}, \\
\left(\Phi_{\tilde{J}} \tilde{g}\right)\left(X^{h}, Y^{v}, Z^{h}\right)= & \frac{\sqrt{5}}{2(1+g(u, u))}\left\{g(Y, u) g\left(\nabla_{X} Z, u\right)-g\left(Y, \nabla_{X} Z\right) g(u, u)\right\}, \\
\left(\Phi_{\tilde{J}} \tilde{g}\right)\left(X^{v}, Y^{h}, Z^{h}\right)= & 0, \\
\left(\Phi_{\tilde{J}} \tilde{g}\right)\left(X^{h}, Y^{v}, Z^{v}\right)= & \frac{\sqrt{5}}{2(1+g(u, u))^{2}}\{-2 g(Y, Z) g(X, u)+g(X, Y) g(Z, u) \\
& +g(X, Z) g(Y, u)\}, \\
\left(\Phi_{\tilde{J}} \tilde{g}\right)\left(X^{v}, Y^{h}, Z^{v}\right)= & -\frac{\sqrt{5}}{2(1+g(u, u))^{2}}\{-2 g(Y, Z) g(X, u)+g(X, Y) g(Z, u) \\
& +g(X, Z) g(Y, u)+g(R(Y, X) u, Z) \\
& +g(R(Y, X) u, Z) g(u, u)\}, \\
\left(\Phi_{\tilde{J}} \tilde{g}\right)\left(X^{v}, Y^{v}, Z^{h}\right)= & -\frac{\sqrt{5}}{2(1+g(u, u))} g(Y, R(Z, X) u), \\
\left(\Phi_{\tilde{J}} \tilde{g}\right)\left(X^{v}, Y^{v}, Z^{v}\right)= & 0 .
\end{aligned}
$$

Thus, from Theorem 1 and Theorem 2, if $g(X, Y) u+g(Y, u) X=2 g(X, u) Y$ holds on $M$ for all vector fields $X$ and $Y$, then $\left(\Phi_{\tilde{J}} \tilde{g}\right)\left(X^{i}, Y^{j}, Z^{k}\right)$ vanishes for all $i, j, k \in$ $\{h, v\}$ and this completes the proof.

\section{REFERENCES}

[1] M. Abbassi and M. Sarih, "Killing vector fields on tangent bundles with Cheeger-Gromoll metric," Tsukuba J. Math., vol. 27, no. 2, pp. 295-306, 2003. 
[2] J. Cheeger and D. Gromoll, "On the structure of complete manifolds of non-negative curvature," Ann. of Math., vol. 96, pp. 413-443, 1972, doi: 10.2307/1970819.

[3] P. Dombrowski, "On the geometry of the tangent bundle," J. Reine Angew. Math., vol. 210, pp. 73-88, 1962, doi: 10.1515/crll.1962.210.73.

[4] A. Gezer, N. Cengiz, and A. Salimov, "On integrability of golden Riemannian structures," Turk J Math., vol. 37, pp. 693-703, 2013, doi: 10.3906/mat-1108-35.

[5] S. Gudmundsson and E. Kappos, "On the geometry of tangent bundles," Expo. Math., vol. 20, pp. 1-41, 2002, doi: 10.1016/S0723-0869(02)80027-5.

[6] S. Gudmundsson and E. Kappos, "On the geometry of the tangent bundle with the CheegerGromoll metric," Tokyo J. Math., vol. 25, no. 1, pp. 75-83, 2002.

[7] H. Hou and L. Sun, "Geometry of tangent bundle with Cheeger-Gromoll type metric," J. Math. Anal. Appl., vol. 402, pp. 493-504, 2013, doi: 10.1016/j.jmaa.2013.01.043.

[8] C.-E. Hretcanu and M. Crasmareanu, "Metallic structures on Riemannian manifolds," Revista De La Union Matematica Argentina, vol. 54, no. 2, pp. 15-27, 2013.

[9] A. Kazan and H. Karadağ, "Paracontact tangent bundles with Cheeger-Gromoll metric," Mediterr. J. Math., vol. 12, no. 2, pp. 497-523, 2015, doi: 10.1007/s00009-014-0414-1.

[10] O. Kowalski, "Curvature of the induced Riemannian metric of the tangent bundle of Riemannian manifold," J. Reine Angew. Math., vol. 250, pp. 124-129, 1971, doi: 10.1515/crll.1971.250.124.

[11] M. Munteanu, "Cheeger-Gromoll type metrics on the tangent bundle," Sci. Ann. Univ. Agric. Sci. Vet. Med., vol. 49, no. 2, pp. 257-268, 2006.

[12] M. Munteanu, "Some aspects on the geometry of the tangent bundles and tangent sphere bundles of a Riemannian manifold," Mediterr. J. Math., vol. 5, pp. 43-59, 2008, doi: 10.1007/s00009-0080135-4.

[13] E. Musso and F. Tricerri, "Riemannian metrics on tangent bundles," Ann. Mat. Pura Appl., vol. 4, no. 150 , pp. 1-20, 1988, doi: 10.1007/BF01761461.

[14] A. A. Salimov and K. Akbulut, "A note on a paraholomorphic Cheeger-Gromoll metric," Proc. Indian Acad. Sci. (Math. Sci.), vol. 119, no. 2, pp. 187-195, 2009, doi: 10.1007/s12044-009-0019y.

[15] S. Sasaki, "On the differential geometry of tangent bundles of Riemannian manifolds i," Tohoku Math. J., vol. 10, no. 3, pp. 338-354, 1958, doi: 10.2748/tmj/1178244668.

[16] M. Sekizawa, "Curvatures of tangent bundles with Cheeger-Gromoll metric," Tokyo J. Math., vol. 14 , no. 2 , pp. 407-417, 1991, doi: 10.3836/tjm/1270130381.

Authors' addresses

Ahmet Kazan

İnönü University, Department of Mathematics, Faculty of Arts and Sciences, 44280 Malatya, Turkey

E-mail address: ahmet.kazan@inonu.edu.tr

H. Bayram Karadă

İnönü University, Department of Mathematics, Faculty of Arts and Sciences, 44280 Malatya, Turkey

E-mail address: bayram.karadag@inonu.edu.tr 